\title{
PARAMETRIC DOWN-CONVERSION OF HIGHER-ORDER BESSEL OPTICAL BEAM IN CUBIC NONLINEAR MEDIUM
}

\author{
V. Pyragaitė and A. Stabinis \\ Department of Quantum Electronics, Faculty of Physics, Vilnius University, Sauletekio 9, LT-10222 Vilnius, Lithuania \\ E-mail: algirdas.stabinis@ff.vu.lt
}

Received 10 March 2006

\begin{abstract}
It is revealed that the orbital angular momentum is conserved during the spontaneous parametric down-conversion of a higher-order Bessel beam propagating in a cubic nonlinear medium. This is the result of transverse selection of the spontaneously arising optical fields in an optical parametric generator pumped by Bessel beam, due to transverse phase matching of interacting conical waves.
\end{abstract}

Keywords: Bessel beam, parametric down-conversion, orbital angular momentum

PACS: 42.60.Jf, 42.65.-k, 42.65.Yj

\section{Introduction}

Along with the spin angular momentum, the photons can also carry the orbital angular momentum (OAM) [1]. The latter momentum can arise from the helical phase front of the propagating beam. OAM eigenstates have an azimuthal phase dependence of the form $\exp (\mathrm{i} l \psi)$, where $l$ (topological charge) is an integer and corresponds to the number of times the phase changes by $2 \pi$ in a closed loop around the beam. The photons of such a beam carry the OAM of magnitude $l \hbar[2,3]$.

A particular interest is attached to OAM in a spontaneous parametric down-conversion (SPDC) in $\chi^{(2)}$ crystals. It was predicted and experimentally confirmed that SPDC provides an entanglement of OAM photon states, which is related to the conservation of OAM [39]. In the case of classical Laguerre-Gaussian fields the OAM is not conserved as an observable property within SPDC in optical parametric generator (OPG) [10]. In contrary, it was revealed that OAM can be conserved in the SPDC of higher-order Bessel beams [11].

Recently, it was demonstrated that SPDC in $\chi^{(3)}$ medium is also an excellent source of quantumcorrelated photon pairs $[12,13]$. In what follows, we demonstrate that OAM is conserved within the SPDC of higher-order Bessel classical fields in $\chi^{(3)}$ medium based OPG.

The Bessel beam is a conical beam, which can be described as a superposition of plane monochromatic waves of frequency $\omega$ with wave vectors that lie on the surface of a cone (Fig. 1(a)):

$$
\begin{aligned}
f(t, x, y, z) & =\exp \left[\mathrm{i}\left(\omega t-k_{z} z\right)\right] \frac{1}{2 \pi} \\
\times & \int_{0}^{2 \pi} S(\psi) \exp [-\mathrm{i} \beta(x \cos \psi+y \sin \psi)] \mathrm{d} \psi .
\end{aligned}
$$

In this case the magnitudes of transverse $\beta=k \sin \alpha$ and longitudinal $k_{z}=k \cos \alpha$ components of all wave vectors $\mathbf{k}$ are the same. Here $\alpha$ is a half-cone angle. We have $k^{2}=\beta^{2}+k_{z}^{2}, \beta^{2}=k_{x}^{2}+k_{y}^{2}, k_{x}=\beta \cos \psi$, and $k_{y}=\beta \sin \psi$, where $\psi$ is the azimuthal angle. Defining the angles $\theta$ and $\varphi$ as $\tan \theta=k_{x} / k_{z}$ and $\tan \varphi=k_{y} / k_{z}$ in the paraxial approximation we obtain $k_{x} \approx k \theta, k_{y} \approx k \varphi, \beta \approx k \alpha, k_{z} \approx k-k \alpha^{2} / 2$, $\theta \approx \alpha \cos \psi, \varphi \approx \alpha \sin \psi$, and $\theta^{2}+\varphi^{2} \approx \alpha^{2}$. The spatial spectrum of the conical beam is a ring of radius $\alpha$, Fig. 1(b). If $S(\psi)=S_{0} \exp [i \rho(\psi+\pi / 2)]$, where $\rho$ is an integer number, then Eq. (1) describes $\rho$-order Bessel beam (or Bessel vortex beam of topological charge $\rho$ ):

$$
f(t, x, y, z)=S_{0} J_{\rho}(\beta r) \exp \left[\mathrm{i} \rho \vartheta+\mathrm{i}\left(\omega t-k_{z} z\right)\right],
$$

where $r=\sqrt{x^{2}+y^{2}}$ and $\vartheta=\arctan (y / x)$.

We investigate the case when the cubic nonlinear medium is pumped by two higher-order Bessel beams. The output radiation is determined by the phase 

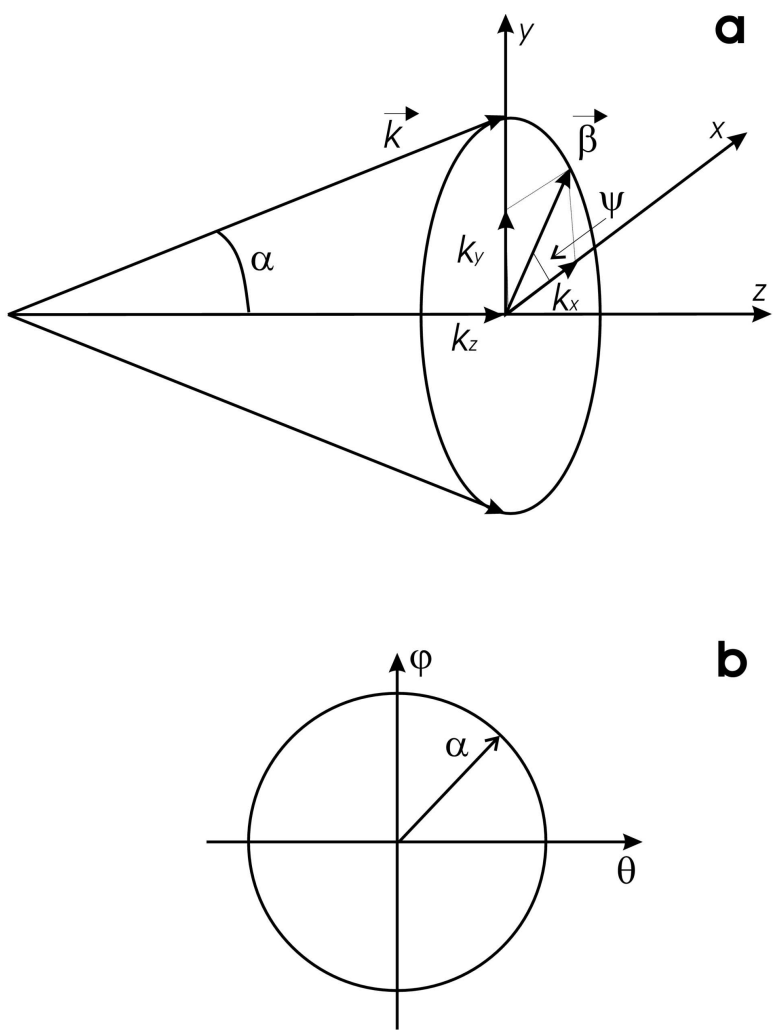

Fig. 1. Spatial spectrum of conical beam (a) in Cartesian coordinates and (b) in transverse $\theta, \varphi$ plane.

matching conditions of conical beams which for fourwave interaction follow from

$$
\begin{aligned}
& k_{1 x}+k_{2 x}=k_{3 x}+k_{4 x}, k_{1 y}+k_{2 y}=k_{3 y}+k_{4 y}, \\
& k_{1 z}+k_{2 z}=k_{3 z}+k_{4 z}, \omega_{1}+\omega_{2}=\omega_{3}+\omega_{4},
\end{aligned}
$$

where subscripts $1,2,3$, and 4 denote the wave vectors and frequencies of signal, idler, and two pump conical beams, respectively. The first two of Eqs. (3) describe the transverse phase matching (TPM) conditions. The third of Eqs. (3) corresponds to the condition of longitudinal phase matching (LPM).

\section{Transverse phase matching}

The first and the second of Eqs. (3) can be rewritten as

$$
\begin{aligned}
& \beta_{1} \cos \phi_{1}+\beta_{2} \cos \phi_{2}=\beta_{3} \cos \phi_{3}+\beta_{4}, \\
& \beta_{1} \sin \phi_{1}+\beta_{2} \sin \phi_{2}=\beta_{3} \sin \phi_{3},
\end{aligned}
$$

where $\phi_{1}=\psi_{1}-\psi_{4}, \phi_{2}=\psi_{2}-\psi_{4}$, and $\phi=\psi_{3}-\psi_{4}$. Obviously, two pump cones at given values $\beta_{3}$ and $\beta_{4}$ can be transversally phase-matched simultaneously with many pairs of signal and idler cones having different values $\beta_{1}$ and $\beta_{2}$. Further we investigate the case when $\omega_{3}=\omega_{4}=\omega_{0}$ and $\beta_{3}=\beta_{4}=\beta_{0}$. It is important that the nonlinear coupling of pump and various signal and idler cones is different and depends on values of $p=\beta_{1} / \beta_{0}$ and $q=\beta_{2} / \beta_{0}$. The magnitude of this coupling can be determined by TPM integral, which describes an overlap of the interacting conical beams. In the case of four-wave interaction the TPM integrals $S_{1}\left(\beta_{1}, \psi\right)$ and $S_{2}\left(\beta_{2}, \psi\right)$ for signal and idler waves, respectively, can be written as

$$
\begin{aligned}
S_{1}\left(\beta_{1}, \psi\right)=\int_{0}^{\infty} \int_{0}^{2 \pi} r A_{2}^{*}(r, \vartheta) A_{3}(r, \vartheta) A_{4}(r, \vartheta) \\
\quad \times \exp \left[\mathrm{i} \beta_{1} r \cos (\psi-\vartheta)\right] \mathrm{d} r \mathrm{~d} \vartheta, \\
S_{2}\left(\beta_{2}, \psi\right)=\int_{0}^{\infty} \int_{0}^{2 \pi} r A_{1}^{*}(r, \vartheta) A_{3}(r, \vartheta) A_{4}(r, \vartheta) \\
\quad \times \exp \left[\mathrm{i} \beta_{2} r \cos (\psi-\vartheta)\right] \mathrm{d} r \mathrm{~d} \vartheta,
\end{aligned}
$$

where $A_{j}(j=1,2,3,4)$ are complex amplitudes of interacting waves in cubic nonlinear medium. In fact, these integrals determine the spatial spectra of signal and idler waves excited by the pump beams from quantum noise level. Further we assume that all interacting waves are apertured Bessel beams (Bessel-Gauss beams), the complex amplitudes of which are

$$
\begin{aligned}
& A_{1}=a_{1} \exp \left(-\frac{r^{2}}{d_{1}^{2}}\right) J_{m}\left(\beta_{1} r\right) \exp (\mathrm{i} m \vartheta), \\
& A_{2}=a_{2} \exp \left(-\frac{r^{2}}{d_{2}^{2}}\right) J_{n}\left(\beta_{2} r\right) \exp (\mathrm{i} n \vartheta), \\
& A_{3}=a_{3} \exp \left(-\frac{r^{2}}{d_{3}^{2}}\right) J_{l_{1}}\left(\beta_{3} r\right) \exp \left(\mathrm{i} l_{1} \vartheta\right), \\
& A_{4}=a_{4} \exp \left(-\frac{r^{2}}{d_{4}^{2}}\right) J_{l_{2}}\left(\beta_{3} r\right) \exp \left(\mathrm{i} l_{2} \vartheta\right),
\end{aligned}
$$

where integer numbers $m, n, l_{1}$, and $l_{2}$ are topological charges of signal, idler, and two pump beams, respectively. Then, integration of Eqs. (5) over $\vartheta$ at $l_{1}+l_{2}=m+n$ gives

$$
\begin{aligned}
S_{1}= & a_{2} a_{3} a_{4} \exp \left[\operatorname{im}\left(\psi+\frac{\pi}{2}\right)\right] \\
\times & \int_{0}^{\infty} r \exp \left(-\frac{r^{2}}{d_{234}^{2}}\right) J_{m}\left(\beta_{1} r\right) J_{n}\left(\beta_{2} r\right) \\
& \times J_{l_{1}}\left(\beta_{3} r\right) J_{l_{2}}\left(\beta_{3} r\right) \mathrm{d} r,
\end{aligned}
$$



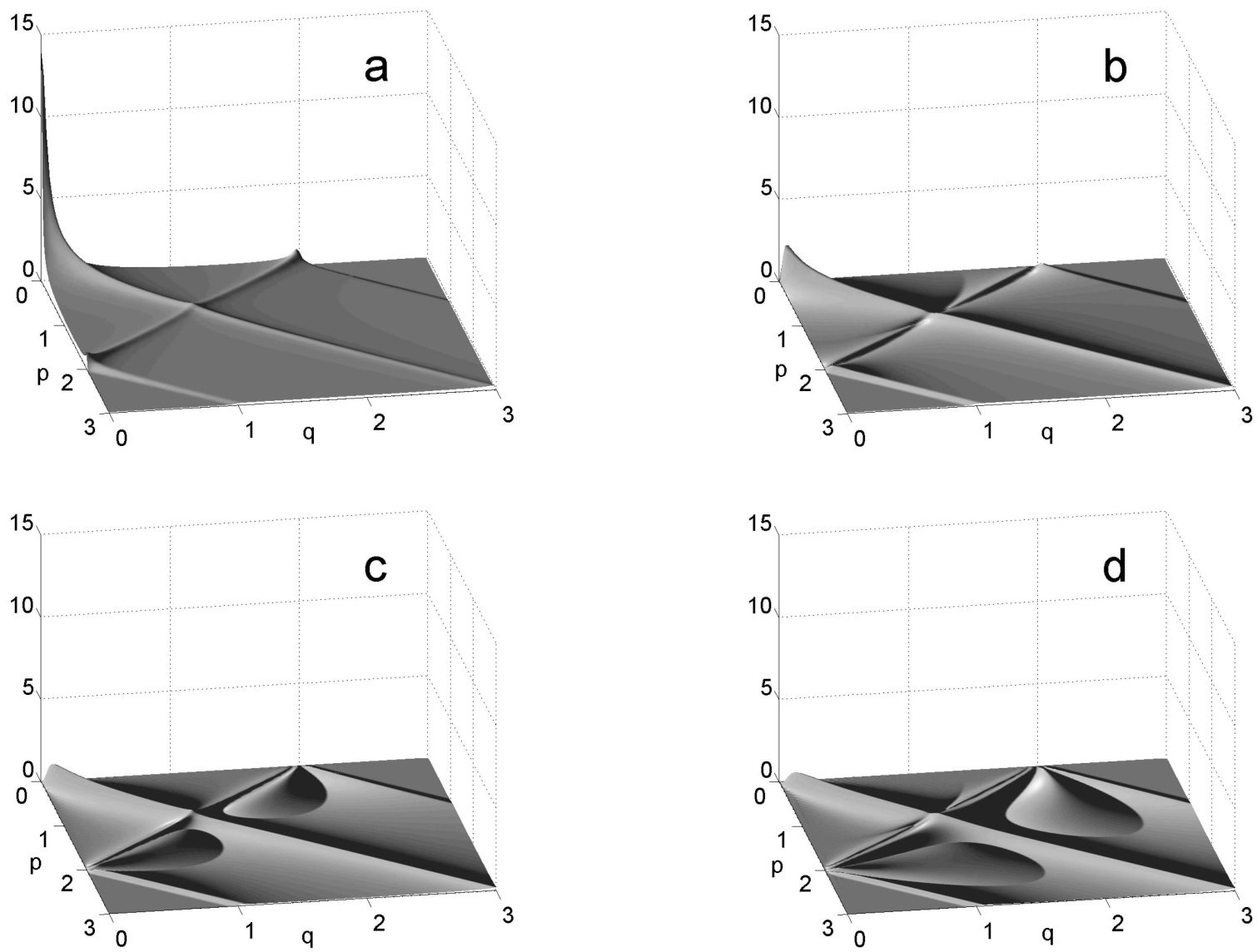

Fig. 2. TPM integral $T_{m n l_{1} l_{2}}(p, q)$ for (a) $l_{1}=l_{2}=0$ and $m=n=0$, (b) $m=-1, n=1$, (c) $m=-2, n=2$, (d) $m=-3, n=3$. $g=50$.

$$
\begin{aligned}
S_{2}= & a_{1} a_{3} a_{4} \exp \left[\mathrm{i} n\left(\psi+\frac{\pi}{2}\right)\right] \\
\times & \int_{0}^{\infty} r \exp \left(-\frac{r^{2}}{d_{134}^{2}}\right) J_{m}\left(\beta_{1} r\right) J_{n}\left(\beta_{2} r\right) \\
& \times J_{l_{1}}\left(\beta_{3} r\right) J_{l_{2}}\left(\beta_{3} r\right) \mathrm{d} r,
\end{aligned}
$$

where $d_{134}^{-2}=d_{1}^{-2}+d_{3}^{-2}+d_{4}^{-2}$ and $d_{234}^{-2}=d_{2}^{-2}+$ $d_{3}^{-2}+d_{4}^{-2}$. We note that for $l_{1}+l_{2} \neq m+n$ TPM integrals $S_{1}$ and $S_{2}$ (Eqs. (5)) are zeroes. So, the signal and idler vortex beams of topological charges $m$ and $n$ interact parametrically with two pump Bessel beams of topological charges $l_{1}$ and $l_{2}$ only if the charge conservation law $l_{1}+l_{2}=m+n$ is fulfilled. In general, the nonlinear coupling of signal, idler, and two pump Bessel beams can be characterized by a common normalized TPM integral

$$
T_{m n l_{1} l_{2}}=\mid \int_{0}^{\infty} x \exp \left(-\frac{x^{2}}{g^{2}}\right) J_{m}(p x) J_{l_{1}+l_{2}-m}(q x)
$$

$$
\times J_{l_{1}}(x) J_{l_{2}}(x) d x \mid,
$$

where, as earlier, $p$ and $q$ are normalized components of transverse wave vectors of the signal and idler waves, and $g=\beta_{0} d_{134}$ (or $\left.\beta_{0} d_{234}\right) \gg 1$.

The numerically calculated integrals $T_{m n l_{1} l_{2}}(p, q)$ for $l_{1}, l_{2}=0,1$ and various values $m$ and $n$ are presented in Figs. 2-5. At $l_{1}=0, l_{2}=0$ (Fig. 2) the absolute maximum is obtained for $p=0$ and $q=0$, when $m=n=0$. Accordingly we have $\beta_{1}=\beta_{2}=0$. Therefore, the strongest nonlinear coupling with two zeroth-order pump beams is obtained when both the signal and idler beams are axial beams. Obviously, the coupling of two zeroth-order pump beams with the signal and idler higher-order Bessel beams of opposite topological charges is rather weak, compare Fig. 2(a) and $(\mathrm{b}-\mathrm{d})$.

At $l_{1}=l_{2}=1$ the strongest coupling is observed when $m=0, n=2$ and $p=0, q \approx 0.08$ (Fig. 3). We have $T_{0211}(p, q)=T_{2011}(q, p)$, so the output radiation will consist of two axial (signal and idler) and two axial 

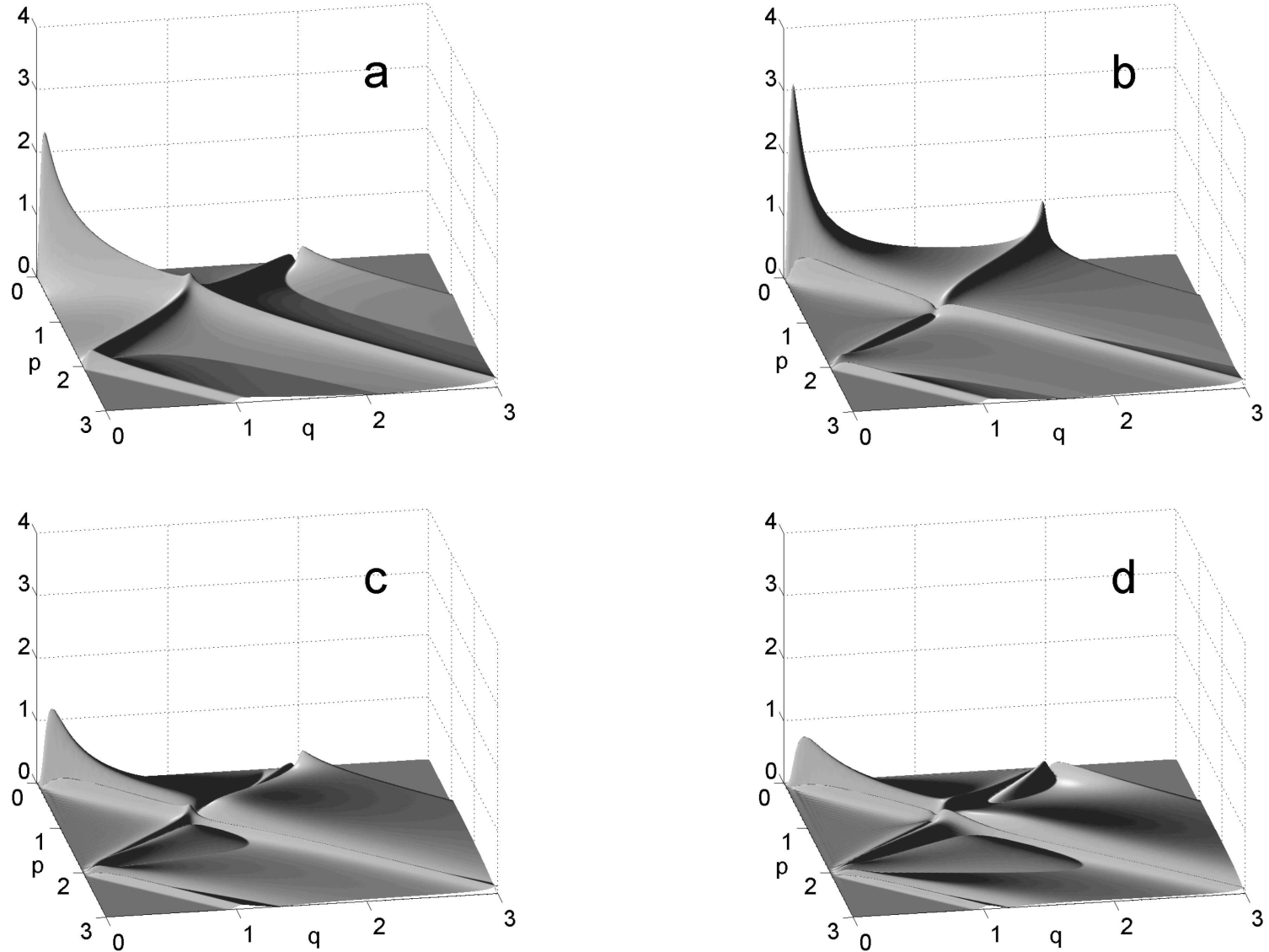

Fig. 3. TPM integral $T_{m n l_{1} l_{2}}(p, q)$ for (a) $l_{1}=l_{2}=1$ and $m=n=1$, (b) $m=0, n=2$, (c) $m=-1, n=3$, (d) $m=-2, n=4$. $g=50$.

vortical (idler and signal) beams. The transverse wave vector of the vortical beam $\beta_{2}$ (or $\beta_{1}$ ) is much smaller than $\beta_{0}$.

At $l_{1}=0, l_{2}=1$ the strongest coupling is observed when $m=0, n=1$ and $p=0, q=2$ (Fig. 4). We have $T_{0101}(p, q)=T_{1001}(q, p)$, so the output radiation consists of two axial (signal and idler) and two conical vortical (idler and signal) beams. The transverse wave vector of the conical beam $\beta_{2}$ (or $\beta_{1}$ ) is equal to $2 \beta_{0}$.

In the case when $l_{1}=1, l_{2}=2$ the strongest coupling is observed when $m=0, n=3$ and $p=0, q=2$ (Fig. 5). We have $T_{0312}(p, q)=T_{3012}(q, p)$, so the output radiation will consist again of two axial (signal and idler) and two conical vortical (idler and signal) beams. The transverse wave vector of the conical beam $\beta_{2}$ (or $\beta_{1}$ ) is equal again to $2 \beta_{0}$.

In general, the best overlap of the four interacting beams is obtained when one (signal or idler) beam is the axial beam possessing zero OAM, and the other (idler or signal) beam is the vortical beam carrying the topological charge $l_{1}+l_{2}$. So, the output radiation will consist of two OAM-free and two vortical beams with OAM equal to the sum of OAM of two pump beams.

\section{Longitudinal phase matching}

The LPM condition allows to determine the frequencies $\omega_{1}$ and $\omega_{2}$ of signal and idler waves. At $\omega_{3}=\omega_{4}$ and $\beta_{3}=\beta_{4}$ we have $k_{3 z}=k_{4 z}$ and the third of Eqs. (3) can be written as

$$
k_{1 z}+k_{2 z}=2 k_{3 z} .
$$

In the paraxial approximation $k_{i z}=k_{i} \cos \left(\alpha_{i}\right) \approx$ $k_{i}\left(1-\alpha_{i}^{2} / 2\right), i=1,2,3$. Then from Eq. (10) it follows that

$$
k_{1}+k_{2}-2 k_{3}=\frac{1}{2} k_{1} \alpha_{1}^{2}+\frac{1}{2} k_{2} \alpha_{2}^{2}-k_{3} \alpha_{3}^{2} .
$$

We suppose that $\omega_{1}=\omega_{0}+\Delta \omega$ and $\omega_{2}=\omega_{0}-\Delta \omega$, where $|\Delta \omega| \ll \omega_{0}$. Then $k\left(\omega_{0} \pm \Delta \omega\right) \approx k_{0} \pm \frac{1}{u_{0}} \Delta \omega+$ $\frac{g_{0}}{2} \Delta \omega^{2}$, where $k_{0}=k\left(\omega_{0}\right), u_{0}=(\partial \omega / \partial k)_{\omega=\omega_{0}}$, and $g_{0}=\left(\partial^{2} k / \partial \omega^{2}\right)_{\omega=\omega_{0}}$. Here $u_{0}$ and $g_{0}$ are group velocity and group velocity dispersion (GVD) coefficient at 

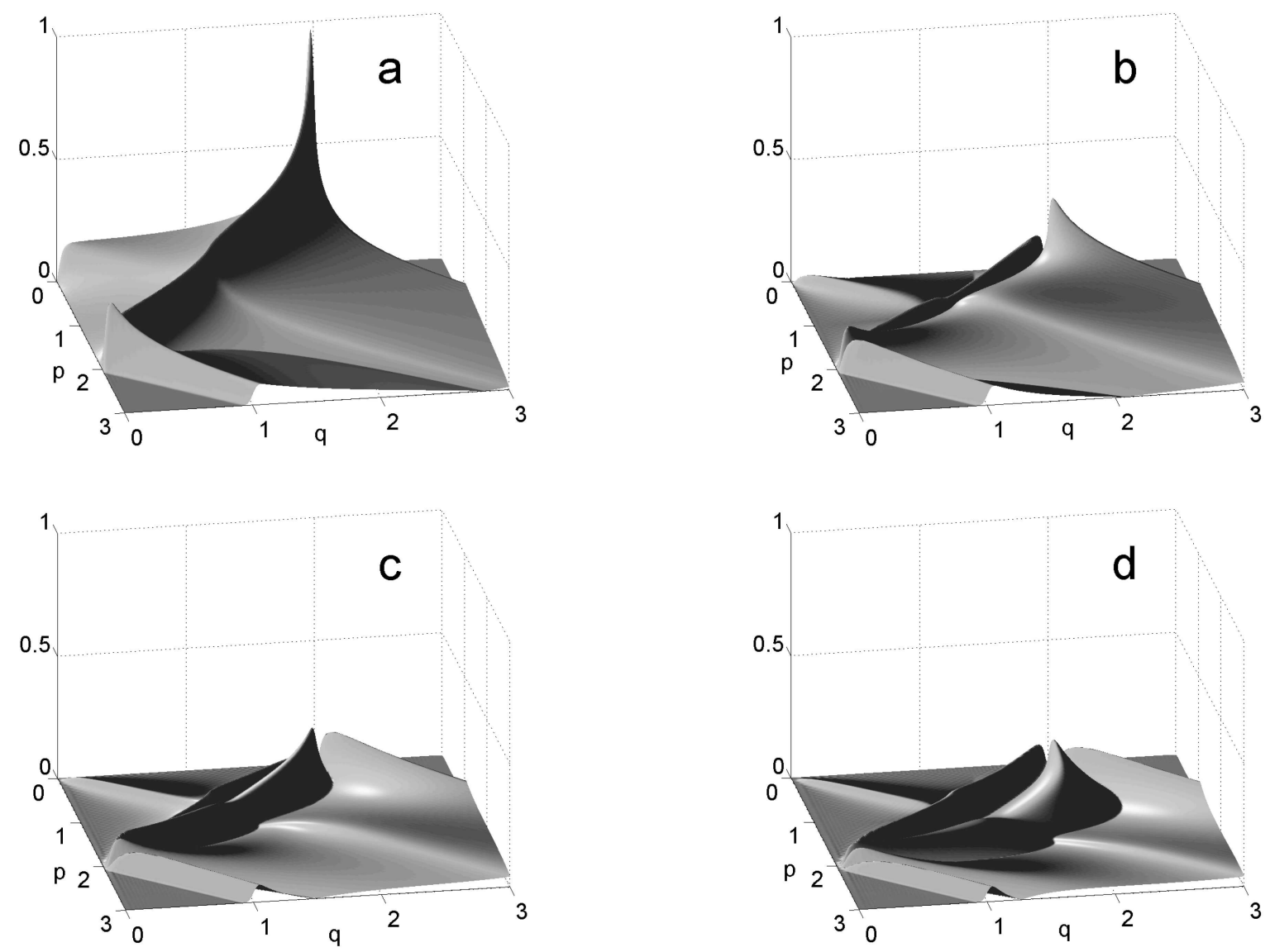

Fig. 4. TPM integral $T_{m n l_{1} l_{2}}(p, q)$ for (a) $l_{1}=0, l_{2}=1$ and $m=0, n=1$, (b) $m=-1, n=2$, (c) $m=-2, n=3$, (d) $m=-3$, $n=4 . g=50$.

$\omega=\omega_{0}$, respectively. Then we can rewrite the Eq. (11) as follows:

$$
g_{0} \Delta \omega^{2} \approx k_{0}\left[\frac{\alpha_{1}^{2}}{2}+\frac{\alpha_{2}^{2}}{2}-\alpha_{3}^{2}\right]
$$

Taking into account that $\alpha_{i} \approx \beta_{i} / k_{0}$ we obtain

$$
\Delta \omega^{2}=\frac{1}{2 k_{0} g}\left(\beta_{1}^{2}+\beta_{2}^{2}-2 \beta_{3}^{2}\right)
$$

Then for $\beta_{1}, \beta_{2} \ll \beta_{3}$ (Figs. 2(a), 3(b)) we have $\omega_{1}=$ $\omega_{0}+\Delta \omega$ and $\omega_{2}=\omega_{0}-\Delta \omega$, where

$$
\Delta \omega^{2}=-\frac{k_{0} \alpha_{3}^{2}}{g_{0}} .
$$

In this case parametric down-conversion is possible in cubic medium with negative GVD coefficient $\left(g_{0}<0\right)$. For $\beta_{1}=0, \beta_{2}=2 \beta_{3}$ or $\beta_{1}=2 \beta_{3}, \beta_{2}=0$ (Figs. 4(a), 5(b)) we obtain

$$
\Delta \omega^{2}=\frac{k_{0} \alpha_{3}^{2}}{g_{0}},
$$

and parametric down-conversion is feasible in cubic nonlinear medium with $g_{0}>0$.

\section{Conclusions}

It is demonstrated that the output radiation of $\chi^{(3)}$ medium-based OPG pumped by a higher-order Bessel beam consists of two OAM-free and two vortical beams the OAM of which are equal to the sum of OAM of two pump beams. So, the OAM is conserved in cubic nonlinear medium within the SPDC of classical higherorder Bessel beam similarly as in the case of quantum fields. That is the result of strong transverse selection of spontaneously arising fields in OPG pumped by Bessel beams. The appearance of conical fields within the SPDC in cubic nonlinear medium is feasible only in the medium with positive GVD coefficient for signal and idler waves. 

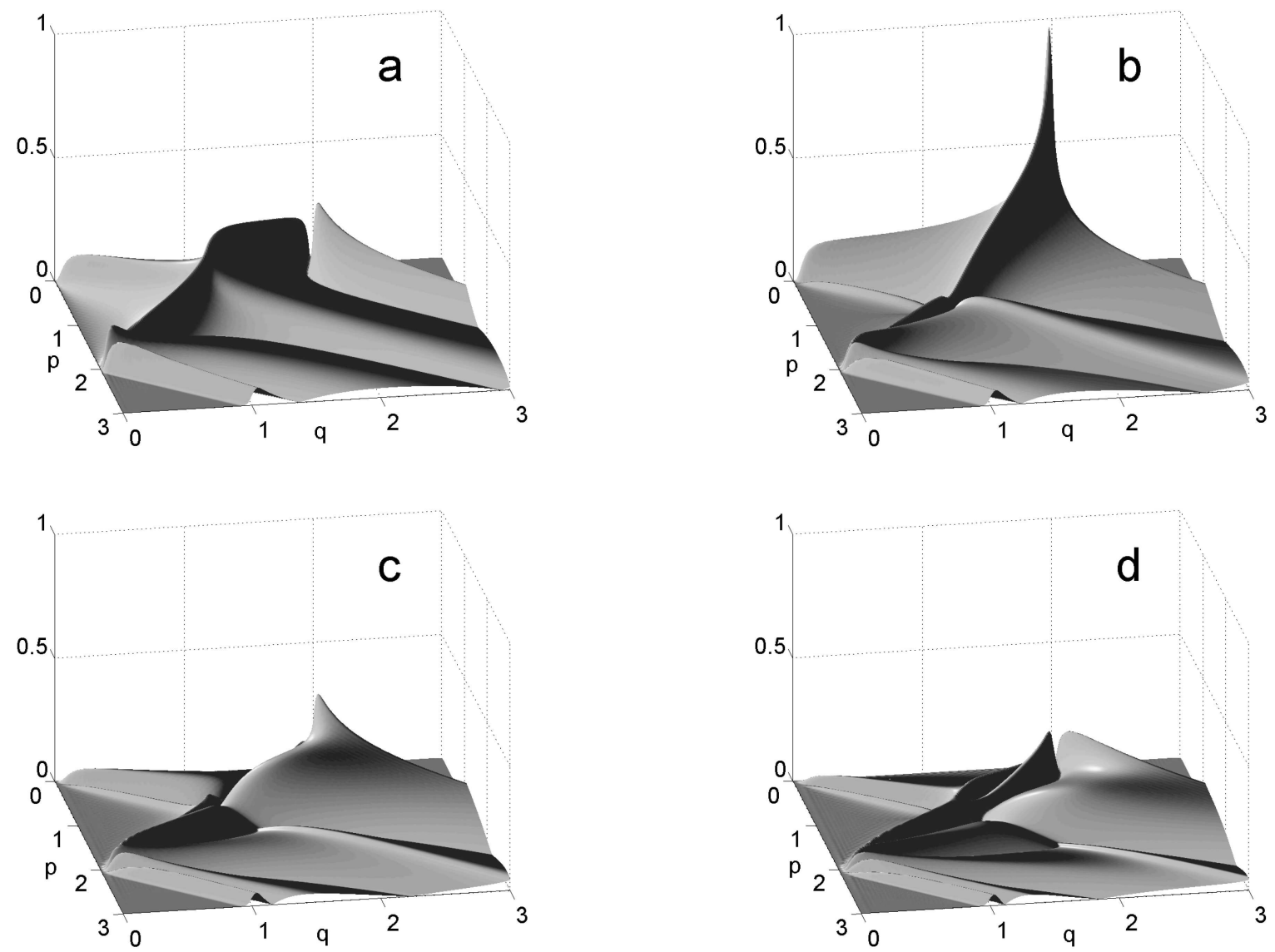

Fig. 5. TPM integral $T_{m n l_{1} l_{2}}(p, q)$ for (a) $l_{1}=1, l_{2}=2$ and $m=1, n=2$, (b) $m=0, n=3$, (c) $m=-1, n=4$, (d) $m=-2, n=5$. $g=50$.

\section{References}

[1] L. Allen, M.J. Padget, and M. Babiker, in Progress in Optics XXXIX, ed. E. Wolf (Elsevier Science, New York, 1999), pp. 291-372.

[2] L. Allen, M.W. Beijersbergen, R.J.C. Spreeuw, and J.P. Woerdman, Orbital angular momentum of light and the transformation of Laguerre-Gaussian laser modes, Phys. Rev. A 45, 8185-8189 (1992).

[3] A. Mair, A. Vaziri, G. Weihs, and A. Zeilinger, Entanglement of the orbital angular momentum states of photons, Nature (London) 412, 313-316 (2001).

[4] M.M. Arnaut and G.A. Barbosa, Orbital and intrinsic angular momentum of single photons and entangled pairs of photons generated by parametric downconversion, Phys. Rev. Lett. 85, 286-289 (2000).

[5] A. Vaziri, G. Weihs, and A. Zeilinger, Experimental two-photon, three-dimensional entanglement for quantum communication, Phys. Rev. Lett. 89, 240401 (2002).

[6] S. Franke-Arnold, S.M. Barnett, M.J. Padgett, and L. Allen, Two-photon entanglement of orbital angular momentum states, Phys. Rev. A 65, 033823 (2002).
[7] G. Molina-Terriza, J.R. Torres, and L. Torner, Orbital angular momentum of photons in noncollinear parametric downconversion, Opt. Commun. 228, 155-160 (2003).

[8] A. Peer, B. Dayan, A.A. Friesen, and Y. Silberberg, Temporal shaping of entangled photons, Phys. Rev. Lett. 94, 073601 (2005).

[9] O. Kuzucu, M. Fiorentino, M.A. Albota, F.N.C. Wong, and F.X. Kaertner, Two-photon coincident frequency entanglement via extended phase matching, Phys. Rev. Lett. 94, 083601 (2005).

[10] J. Arlt, K. Dholakia, L. Allen, and M.J. Padgett, Parametric down-conversion for light beams possessing orbital angular momentum, Phys. Rev. A 59, 3950-3952 (1999).

[11] V. Pyragaite, A. Piskarskas, K. Regelskis, V. Smilgevicius, A. Stabinis, and S. Mikalauskas, Parametric down-conversion of higher-order Bessel optical beams in quadratic nonlinear medium, Opt. Commun. 240, 191-200 (2004).

[12] X. Li, J. Chen, P. Voss, J. Sharping, and P. Kumar, Allfiber photon-pair source for quantum communications: Improved generation of correlated photons, Opt. Express 12, 3737-3744 (2004). 
[13] X. Li, P.L. Voss, J.E. Sharping, and P. Kumar, Opticalfiber source of polarization-entangled photons in the
$1550 \mathrm{~nm}$ telecom band, Phys. Rev. Lett. 94, 053601 (2005).

\title{
PARAMETRINIS AUKŠTESNĖS EILĖS BESELIO OPTINIO PLUOŠTO DAŽNIO MAŽINIMAS KUBINĖJE NETIESINĖJE TERPĖJE
}

\author{
V. Pyragaitè, A. Stabinis
}

Vilniaus universitetas, Vilnius, Lietuva

\section{Santrauka}

Parodyta, kad savaiminio parametrinio aukštesnès eilès Beselio (Bessel) pluošto dažnio mažinimo kubinèje netiesinèje terpèje metu galioja orbitinio kampinio momento tvermès dèsnis. Tai yra parametriniame šviesos generatoriuje vykstančios optinių laukų skersinès atrankos pasekmè, kai generatorius kaupinamas Beselio pluoštu. 